Khalid K. Ali*, Mohamed A. Abd El salam and Emad M. H. Mohamed

\title{
A numerical technique for a general form of nonlinear fractional-order differential equations with the linear functional argument
}

https://doi.org/10.1515/ijnsns-2019-0281

Received November 12, 2019; accepted July 23, 2020; published online September 17, 2020

\begin{abstract}
In this paper, a numerical technique for a general form of nonlinear fractional-order differential equations with a linear functional argument using Chebyshev series is presented. The proposed equation with its linear functional argument represents a general form of delay and advanced nonlinear fractional-order differential equations. The spectral collocation method is extended to study this problem as a discretization scheme, where the fractional derivatives are defined in the Caputo sense. The collocation method transforms the given equation and conditions to algebraic nonlinear systems of equations with unknown Chebyshev coefficients. Additionally, we present a general form of the operational matrix for derivatives. A general form of the operational matrix to derivatives includes the fractionalorder derivatives and the operational matrix of an ordinary derivative as a special case. To the best of our knowledge, there is no other work discussed this point. Numerical examples are given, and the obtained results show that the proposed method is very effective and convenient.
\end{abstract}

Keywords: Caputo fractional derivatives; Chebyshev collocation method; functional argument; nonlinear fractional-order differential equations.

*Corresponding author: Khalid K. Ali, Department of Mathematics, Faculty of Science, Al-Azhar University, Cairo, Egypt, E-mail: khalidkaram2012@azhar.edu.eg, khalidkaram2012@yahoo.com. https://orcid.org/0000-0002-78012760

Mohamed A. Abd El salam and Emad M. H. Mohamed, Department of Mathematics, Faculty of Science, Al-Azhar University, Cairo, Egypt, E-mail: mohamed_salam1985@yahoo.com (M.A. Abd El salam), emad2015201535@gmail.com (E.M.H. Mohamed)

\section{Introduction}

Fractional differential equations have been of great interest for the past three decades. This is due to the intensive development of the theory of fractional calculus itself, as well as its applications. Apart from diverse areas of pure mathematics, fractional differential equations can be used in modeling of various fields of science and engineering such as rheology, self-similar dynamical processes, porous media, fluid flows, viscoelasticity, electrochemistry, control, electromagnetic and many other branches of science [1-5]. Delay differential equations were initially introduced in the 18th century by Laplace and Condorect [6]. However, the rapid development of the theory and applications of those equations did not come until after the Second World War and continues till today. Additionally, the numerical solution of delay and advanced differential equations of fractional order have been reported by many researchers [7-15]. Differential equations of advanced argument had fewer contributions in mathematics research, compared to delay differential equations, which had a great development in the last decade [16-20]. The equations with functional form of argument represent mixed type equations delay, proportional delay and advanced differential equation. All reported works considered a generalization of equations with functional argument with integer-order derivative or with fractional derivative in the linear case.

In this work, we introduce a general form of nonlinear fractional-order differential equations (GNFDEs) with linear functional argument.

$$
\begin{aligned}
& \sum_{k=0}^{n_{1}} \sum_{i=0}^{n_{2}} Q_{k, i}(x) y^{k}(x) y^{\left(v_{i}\right)}\left(p_{i} x+\xi_{i}\right)+ \\
& \sum_{h=1}^{n_{3}} \sum_{j=0}^{n_{4}} P_{h, j}(x) y^{(h)}(x) y^{\left(\alpha_{j}\right)}\left(q_{j} x+\zeta_{j}\right)=f(x),
\end{aligned}
$$

where $x \in[a, b], Q_{k, i}(x), P_{h, j}(x), f(x)$ are well-defined known functions, $a, b, p_{i}, \xi_{i}, q_{j}, \zeta_{j} \in \Re$, where $p_{i}, q_{j} \neq 0, v_{i} \geq 0, \alpha_{j} \geq 0$ and $i-1<v_{i} \leq i, j-1<\alpha_{j} \leq j, n_{i} \in \mathbb{N}$, under the conditions

$$
y^{(i)}\left(\eta_{i}\right)=\mu_{i}, \quad i=0,1,2 \ldots, m-1,
$$

where $\eta_{i} \in[a, b]$ and $m$ is the greatest integer-order derivative existing or the highest integer order greater than 
the fractional derivative. The GNFDE (1) contains at least three different arguments, and then, the following corollary defines the interval that the independent variable $x$ belongs. Chebyshev polynomials of the first kind are used here in this work to approximate the solution of proposed Equation (1). The Chebyshev polynomials are defined on $[-1,1]$.

Corollary 1.1. The independent variable $x$ of (1) belongs to $[a, b]$, which is the intersection of the intervals of the different arguments and $[-1,1]$,

$$
\text { i.e. } x \in[a, b]=\left[\frac{-1+\zeta_{i}}{p_{i}}, \frac{1+\xi_{i}}{p_{i}}\right] \cap\left[\frac{-1+\zeta_{j}}{q_{j}}, \frac{1+\zeta_{j}}{q_{j}}\right] \cap[-1,1]
$$

\section{General notations}

In this section, some definitions and properties for the fractional derivative are listed.

\subsection{The fractional derivative definition}

The Caputo fractional derivative operator $D^{v}$ of order $v$ is defined in the following form:

$$
D^{\nu} G(x)=\frac{1}{\Gamma(n-v)} \int_{0}^{x} \frac{G^{(n)}(t)}{(x-t)^{v-n+1}} d t, \quad v>0,
$$

where $x>0, n-1<v \leq n, n \in \mathbb{N}_{0}$, and $\mathbb{N}_{0}=\mathbb{N}-\{0\}$.

- $D^{v} \sum_{i=0}^{m} \lambda_{i} G_{i}(x)=\sum_{i=0}^{m} \lambda_{i} D^{v} G_{i}(x)$, where $\lambda_{i}$ and $v$ are constants.

- The Caputo fractional differentiation of a constant is zero.

- $\quad D^{v} x^{k}=\left\{\begin{array}{ll}0, & \text { for } k \in \mathbb{N}_{0} \text { and } k<\lceil v\rceil \\ \frac{\Gamma(k+1) x^{k-v}}{\Gamma(k+1-v)}, & \text { for } k \in \mathbb{N}_{0} \text { and } k \geq\lceil\nu\rceil\end{array}\right.$,

where $[v]$ denotes to the smallest integer greater than or equal to $v$.

\section{Procedure solution using the collocation method}

The solution $y(x)$ of (1) may be expanded by Chebyshev polynomials series of the first kind as follows [21]:

$$
y(x)=\sum_{n=0}^{\infty} c_{n} T_{n}(x)
$$

by truncate the series (4) to $N$, where $N<\infty$, and then, the approximate solution is expressed in the following form:

$$
\begin{aligned}
y(x) & \cong \sum_{n=0}^{N} c_{n} T_{n}(x) \\
& =T(x) C,
\end{aligned}
$$

where $T(x)$ and $C$ are matrices given by the following equation:

$$
T(x)=\left[T_{0}(x) T_{1}(x) \ldots T_{N}(x)\right], \quad C=\left[\frac{1}{2} c_{0}, c_{1}, c_{2}, \ldots, c_{N}\right]^{T} .
$$

Now, relation (5) may written in the following form:

$$
y(x)=X(x) W^{T} C,
$$

where $W$ is square lower triangle matrix with size $(N+1) \times(N+1)$ given by the following equation:

$$
W_{i j}= \begin{cases}1, & \text { if } i=j=0 \\
(-1)^{k} 2^{i-2 k} \frac{i}{i-k}\left(\begin{array}{c}
i-k \\
k
\end{array}\right), & \text { if } i+j \text { even and } j \leq i, \\
0, & \text { if } j>i, i+j \text { odd. }\end{cases}
$$

where

$$
k=\left\{\begin{array}{cc}
\frac{i}{2}, \ldots, 1,0, & \text { for even } i \\
\frac{i-1}{2}, \ldots, 1,0, & \text { for odd } i
\end{array}\right.
$$

For example, if $N=4$ and $N=5$,

$$
\begin{gathered}
W=\left(\begin{array}{ccccc}
1 & 0 & 0 & 0 & 0 \\
0 & 1 & 0 & 0 & 0 \\
-1 & 0 & 2 & 0 & 0 \\
0 & -3 & 0 & 4 & 0 \\
1 & 0 & -8 & 0 & 8
\end{array}\right)_{N=4}, \\
W=\left(\begin{array}{cccccc}
1 & 0 & 0 & 0 & 0 & 0 \\
0 & 1 & 0 & 0 & 0 & 0 \\
-1 & 0 & 2 & 0 & 0 & 0 \\
0 & -3 & 0 & 4 & 0 & 0 \\
1 & 0 & -8 & 0 & 8 & 0 \\
0 & 5 & 0 & -20 & 0 & 16
\end{array}\right)_{N=5} .
\end{gathered}
$$

Then, by substituting from (5) in (1), we get the following equation:

$$
\begin{aligned}
& \sum_{k=0}^{n_{1}} \sum_{i=0}^{n_{2}} Q_{k, r}(x)(T(x) C)^{k} D^{v_{i}} T\left(p_{i} x+\xi_{i}\right) C+ \\
& \sum_{k=1}^{n_{3}} \sum_{i=0}^{n_{4}} P_{h, j}(x)\left(T^{(h)}(x) C\right) D^{\alpha j} T\left(q_{j} x+\zeta_{j}\right) C=f(x),
\end{aligned}
$$

we can write (8) as follows:

$$
\begin{aligned}
& {\left[\sum_{k=0}^{n_{1}} \sum_{i=0}^{n_{2}} Q_{k, r}(x)(T(x) C)^{k} D^{v_{i}} T\left(p_{i} x+\xi_{i}\right)+\right.} \\
& \left.\sum_{h=1}^{n_{4}} \sum_{j=0}^{n_{4}} P_{h, j}(x)\left(T^{(h)}(x) C\right) D^{\alpha_{j}} T\left(q_{j} x+\zeta_{j}\right)\right] C=f(x),
\end{aligned}
$$

the collocation points is defined in the following form: 


$$
x_{l}=l h+a
$$

where

$$
h=\frac{b-a}{N}, \quad l=0,1,2, \ldots, N .
$$

By substituting the collocation points (10) in (9), we get the following equation:

$$
\begin{aligned}
& {\left[\sum_{k=0}^{n_{1}} \sum_{i=0}^{n_{2}} Q_{k, i}\left(x_{l}\right)\left(T\left(x_{l}\right) C\right)^{k} D^{v_{i}} T\left(p_{i} x+\zeta_{i}\right)+\right.} \\
& \left.\sum_{h=1}^{n_{4}} \sum_{j=0}^{n_{4}} P_{h, j}\left(x_{l}\right)\left(T^{(h)}\left(x_{l}\right) C\right) D^{\alpha_{j}} T\left(q_{j} x+\zeta_{j}\right)\right] C=f\left(x_{l}\right) .
\end{aligned}
$$

In the following theorem, we introduce a general form of operational matrix of the row vector $T(x)$ in the representation as (6), such that the process includes the fractional-order derivatives, and ordinary operational matrix given as a special case when $\alpha_{i} \rightarrow\left\lceil\alpha_{i}\right\rceil$.

Theorem 1. Assume that, the Chebyshev row vector $T(x)$ is represented as (6), then the order fractional derivative of the vector $D^{\alpha_{i}} T(x)$ is given as follows:

$$
D^{\alpha_{i}} T(x)=X_{\alpha_{i}}(x) B_{\alpha_{i}} W^{T},
$$

where

$$
X_{\alpha_{i}}(x)=\left[x^{-\alpha_{i}+i} x^{1-\alpha_{i}+i} x^{2-\alpha_{i}+i} \ldots x^{N-1-\alpha_{i}+i}\right], \quad i-1<\alpha_{i} \leqslant i,
$$

where $B_{\alpha i}$ is $(N+1) \times(N+1)$ square upper diagonal matrix, and the elements $b_{r, s}$ of $B_{\alpha i}$ can be written as follows:

$$
\left\{\begin{array}{ll}
b_{r, r+i}=\frac{\Gamma(r+i+1)}{\Gamma\left(r+i-\alpha_{i}\right)} & r, s=0,1,2, \ldots, N \\
0 & \text { otherwise }
\end{array},\right.
$$

where $i-1<\alpha_{i} \leqslant i, N \geqslant \alpha_{i}$.

Proof. since

$$
\begin{aligned}
& D^{\alpha_{i}} T(x)=D^{\alpha_{i}}\left[\begin{array}{llll}
1 & x x^{2} & \ldots & x^{N}
\end{array}\right] W^{T}, \\
& =X_{\alpha_{i}} B_{\alpha_{i}} W^{T} \text {, }
\end{aligned}
$$

if $0<\alpha_{1} \leqslant 1$, using Caputo's fractional properties, then we get

$$
\begin{gathered}
X_{\alpha_{1}}=\left[x^{1-\alpha_{1}} \chi^{2-\alpha_{1}} x^{3-\alpha_{1}} \ldots x^{N+1-\alpha_{1}}\right], \\
B_{\alpha_{1}}=\left(\begin{array}{cccc}
0 & \frac{2}{\left.\Gamma 2-\alpha_{1}\right)} & 0 \ldots & 0 \\
0 & 0 & \frac{\Gamma(3)}{\Gamma\left(3-\alpha_{1}\right)} \ldots & 0 \\
\vdots & \vdots & \vdots & \vdots \\
0 & 0 & 0 . . & \frac{\Gamma(N)}{\Gamma\left(N-\alpha_{1}\right)} \\
0 & 0 & 0 . . & 0
\end{array}\right),
\end{gathered}
$$

as $\alpha_{1} \rightarrow 1$, the system reduced to the ordinary case $\left(B_{\alpha 1} \rightarrow B\right)$, see [21].

Also $1<\alpha_{2} \leqslant 2$, then

$$
X_{\alpha_{2}}=\left[\begin{array}{lllll}
x^{2-\alpha_{2}} & x^{3-\alpha_{2}} & \chi^{4-\alpha_{2}} & \ldots & x^{N+2-\alpha_{2}}
\end{array}\right]
$$

$$
B_{\alpha_{2}}=\left(\begin{array}{ccccc}
0 & 0 & \frac{3}{\left(\Gamma 3-\alpha_{2}\right)} \ldots & 0 & 0 \\
0 & 0 & 0 & \frac{\Gamma(4)}{\Gamma\left(4-\alpha_{2}\right)} \ldots & 0 \\
\vdots & \vdots & \vdots & \vdots & \vdots \\
0 & 0 & 0 . . & 0 & \frac{\Gamma(N)}{\Gamma\left(N-\alpha_{2}\right)} \\
0 & 0 & 0 . . & 0 & 0 \\
0 & 0 & 0 . . & 0 & 0
\end{array}\right) \text {, }
$$

as $\alpha_{2} \rightarrow 2$, the system reduced to the ordinary case $\left(B_{\alpha 2} \rightarrow B^{2}\right)$, see [21]

By the same way, if we take $2<\alpha_{3} \leqslant 3$, then

$$
X_{\alpha_{3}}=\left[\begin{array}{lllll}
x^{3-\alpha_{3}} & x^{4-\alpha_{3}} x^{5-\alpha_{3}} \ldots x^{N+3-\alpha_{3}}
\end{array}\right] \text {, }
$$

$B_{\alpha_{3}}=\left(\begin{array}{cccccc}0 & 0 & 0 & \frac{4}{\left(\Gamma 4-\alpha_{3}\right)} \ldots & 0 & 0 \\ 0 & 0 & 0 & 0 & \frac{\Gamma(5)}{\Gamma\left(5-\alpha_{3}\right)} & 0 \\ \vdots & \vdots & \vdots & \vdots & \vdots & \vdots \\ 0 & 0 & 0 . . & 0 & 0 & \frac{\Gamma(N)}{\Gamma\left(N-\alpha_{3}\right)} \\ 0 & 0 & 0 . . & 0 & 0 & 0 \\ 0 & 0 & 0 . . & 0 & 0 & 0 \\ 0 & 0 & 0 . . & 0 & 0 & 0\end{array}\right)$

as $\alpha_{3} \rightarrow 3$, the system reduced to the ordinary case $\left(B_{\alpha 3} \rightarrow B^{3}\right)$, see [21]. By induction, $i-1<\alpha_{i} \leqslant i$, then

$$
X_{\alpha_{i}}(x)=\left[x^{-\alpha_{i}+i} x^{1-\alpha_{i}+i} x^{2-\alpha_{i}+i} \ldots x^{N-1-\alpha_{i}+i}\right], i-1<\alpha_{i} \leqslant i,
$$

and $B_{\alpha i}$ as in (14), where the proposed operational matrix represents a kind of unification of ordinary and fractional case.

Now, we give the matrices representation for all terms in (11) as the representation (12).

*The first nonlinear term in (11) can be written as follows:

$$
\begin{aligned}
& \sum_{k=0}^{n_{1}} \sum_{i=0}^{n_{2}} Q_{k, i}\left(x_{l}\right)\left(T\left(x_{l}\right) C\right)^{k} D^{v_{i}} T\left(p_{i} x_{l}+\xi_{i}\right)= \\
& \sum_{k=0}^{n_{1}} \sum_{i=0}^{n_{2}} Q_{k, i}\left(x_{l}\right)\left(\bar{X} \bar{W}^{T} \bar{C}\right)^{k} X_{v_{i}} B_{v_{i}} H_{p_{i}} E_{\xi_{i}} W^{T} C,
\end{aligned}
$$


where

$$
\begin{aligned}
\bar{X} & =\left(\begin{array}{cccc}
X\left(x_{0}\right) & 0 & 0 \ldots & 0 \\
0 & X\left(x_{1}\right) & 0 \ldots & 0 \\
0 & 0 & X\left(x_{2}\right) \ldots & 0 \\
\vdots & \vdots & \vdots & \vdots \\
0 & 0 & 0 \ldots & X\left(x_{N}\right)
\end{array}\right), \bar{W}^{T}=\left(\begin{array}{cccc}
W^{T} & 0 & 0 \ldots & 0 \\
0 & W^{T} & 0 \ldots & 0 \\
0 & 0 & W^{T} \ldots & 0 \\
\vdots & \vdots & \vdots & \vdots \\
0 & 0 & 0 \ldots & W^{T}
\end{array}\right), \\
\bar{C} & =\left(\begin{array}{cccc}
C & 0 & 0 \ldots & 0 \\
0 & C & 0 \ldots & 0 \\
0 & 0 & C \ldots & 0 \\
\vdots & \vdots & \vdots & \vdots \\
0 & 0 & 0 \ldots & C
\end{array}\right) .
\end{aligned}
$$

and $H_{p i}$ is the square diagonal matrix of the coefficients for the linear argument, and the elements of $H_{p i}$ can be written as follows:

$$
h_{r s}=\left\{\begin{array}{ccc}
0 & \text { if } & r \neq s \\
p_{i}^{r} & \text { if } & r=s
\end{array}\right.
$$

And $E_{\xi i}$ is the square upper triangle matrix for the shift of the linear argument, and the form of $E_{\xi i}$ is given as follows:

$$
E_{\xi_{i}}=\left(\begin{array}{cccc}
\left(\begin{array}{l}
0 \\
0
\end{array}\right)\left(\xi_{i}\right)^{0} & \left(\begin{array}{l}
1 \\
0
\end{array}\right)\left(\xi_{i}\right)^{1-0} & \left(\begin{array}{l}
2 \\
0
\end{array}\right)\left(\xi_{i}\right)^{2-0} \cdots & \left(\begin{array}{c}
N \\
0
\end{array}\right)\left(\xi_{i}\right)^{N-0} \\
0 & \left(\begin{array}{l}
1 \\
1
\end{array}\right)\left(\xi_{i}\right)^{1-1} & \left(\begin{array}{l}
2 \\
1
\end{array}\right)\left(\xi_{i}\right)^{2-1} \ldots & \left(\begin{array}{c}
N \\
1
\end{array}\right)\left(\xi_{i}\right)^{N-1} \\
0 & 0 & \left(\begin{array}{l}
2 \\
2
\end{array}\right)\left(\xi_{i}\right)^{2-2} \cdots & \left(\begin{array}{c}
N \\
2
\end{array}\right)\left(\xi_{i}\right)^{N-2} \\
\vdots & \vdots & \vdots & \vdots \\
0 & 0 & 0 & \cdots\left(\begin{array}{c}
N \\
N
\end{array}\right)\left(\xi_{i}\right)^{N-N}
\end{array}\right) .
$$

*The second nonlinear term in (11) can be written as follows:

$$
\begin{aligned}
& \sum_{h=1}^{n_{3}} \sum_{j=0}^{n_{4}} P_{h, j}\left(x_{l}\right)\left(T^{(h)}\left(x_{l}\right) C\right) D^{\alpha_{j}} T\left(q_{j} x_{l}+\zeta_{j}\right)= \\
& \sum_{h=1}^{n_{3}} \sum_{j=0}^{n_{4}} P_{h, j}\left(x_{l}\right)\left(\overline{X B}_{h} \bar{W}^{T} \bar{C}\right) X_{\alpha_{j}} B_{\alpha_{j}} H_{P i} E_{\zeta j} W^{T} C,
\end{aligned}
$$

where

$$
\bar{B}_{h}=\left(\begin{array}{cccc}
0 & B_{h} & 0 \ldots & 0 \\
0 & 0 & B_{h} \ldots & 0 \\
\vdots & \vdots & \vdots & \vdots \\
0 & 0 & 0 . . & B_{h} \\
0 & 0 & 0 . . & 0
\end{array}\right),
$$

and $B_{h}$ is the same as $B_{\alpha i}$ when $h=\left\lceil\alpha_{i}\right\rceil$.

The matrix representation for the variable coefficients takes the following form:

$$
Q_{i, j}=\left(\begin{array}{ccccc}
Q_{i, j}\left(x_{1}\right) & 0 & 0 & \ldots & 0 \\
0 & Q_{i, j}\left(x_{2}\right) & 0 & \ldots & 0 \\
\vdots & \vdots & \vdots & \vdots & \vdots \\
0 & 0 & 0 & \ldots & Q_{i, j}\left(x_{N}\right)
\end{array}\right) .
$$

Now, by substituting from (23), (24) into (11) and then, we have the following fundamental matrix equation:

$$
\begin{aligned}
& {\left[\sum_{k=0}^{n_{1}} \sum_{i=0}^{n_{2}} Q_{k, i}(x)\left(\overline{X W}^{T} \bar{C}\right)^{k} X_{v i} B_{v i} H_{P i} D_{\xi_{i}} W^{T}\right.} \\
& \left.+\sum_{h=1}^{n_{3}} \sum_{j=0}^{n_{4}} P_{h, j}(x)\left(\overline{X B}_{h} \bar{W}^{T} \bar{C}\right) X_{\alpha j} B_{\alpha_{j}} H_{q j} E_{\zeta j} W^{T}\right] C=\mathrm{F} .
\end{aligned}
$$

We can write (25) in the following form:

$$
U C=F, \quad \text { or }[U ; F],
$$

where

$$
\begin{gathered}
U=\sum_{k=0}^{n_{1}} \sum_{i=0}^{n_{2}} Q_{k, i}(x)\left(\overline{X W}^{T} \bar{C}\right)^{k} X_{v i} B_{v i} H_{P i} E_{\xi_{i}} W^{T} \\
+\sum_{h=1}^{n_{3}} \sum_{j=0}^{n_{4}} P_{h, j}(x)\left(\overline{X B}_{h} \bar{W}^{T} \bar{C}\right) X_{\alpha j} B_{\alpha_{j}} H_{q j} E_{\zeta j} W^{T}, \\
F=\left(\begin{array}{c}
f\left(x_{0}\right) \\
f\left(x_{1}\right) \\
\vdots \\
f\left(x_{N}\right)
\end{array}\right)
\end{gathered}
$$

Corollary 3.1. Suppose $k \geqslant 2$, then the matrix representation for the terms free of derivatives in (1), by using (5), we obtain the following term:

$$
y^{k}(x)=y^{k-1}(x) y(x)=\left(X(x) W^{T} C\right)^{k-1} X(x) W^{T} C .
$$

we can obtain the matrix form for (28) by using the collocation points as follows:

$$
y^{k}(x)=\left(\overline{X W}^{T} \bar{C}\right)^{k-1} X W^{T} C .
$$

${ }^{*}$ we can obtain the matrix form for the conditions (2) by using (5) on the form: 


$$
X\left(\eta_{i}\right) B_{i} W^{T} C=\mu_{i}, \quad i=0,1,2 \ldots, m-1
$$

or

$$
M_{i} C=\left[\mu_{i}\right]
$$

where

$$
M_{i}=X\left(\eta_{i}\right) B_{i} W^{T}
$$

Consequently, replacing $m$ rows of the augmented matrix $[U ; F]$ by rows of the matrix $\left[M_{i} ; \mu_{i}\right]$, we have $[\bar{U} ; \bar{F}]$ or

$$
\bar{U} C=\bar{F} .
$$

The system (25), together with conditions, give $(N+1)$ nonlinear algebraic equations which can be solved, for the unknown $c_{n}, n=0,1,2, \ldots, N$. Consequently $y(x)$ given as Equation (5) can be calculated.

\section{Numerical results}

In this section, five examples are given to demonstrate the applicability and the accuracy of the present method. All results are obtained by using Mathematica 7 programming.

Example 1. Consider the following nonlinear fractional differential equation (NFDE) with linear functional argument:

$$
\begin{aligned}
& y^{2}(x) D^{v_{2}} y(x)+y^{2}(x) y(2 x+1)+y^{4}(x)+y^{\prime}(x) D^{\alpha_{2}} y(x) \\
& \quad=f(x), x \in[0,1]
\end{aligned}
$$

The initial conditions are $y(0)=0, y^{\prime}(0)=1$, and the exact solution is $y(x)=x^{2}+x$ at $v_{2}=1.7, \alpha_{2}=1.5$ where $f(x)=2.25676 x^{0.5}(1+2 x)+2.22849 x^{0.3}$ $\left(x+x^{2}\right)^{2}+\left(x+x^{2}\right)^{4}+\left(x+x^{2}\right)^{2}(2+4(1+2 x))$. We apply the suggested method with $N=3$, and by the fundamental matrix equation of the problem defined in (25), we have the following equation:

$$
\begin{aligned}
& {\left[Q_{2,2}\left(\overline{X W}^{T} \bar{C}\right)^{2} X_{v_{2}} B_{v_{2}} W^{T}+Q_{2,0}\left(\overline{X W}^{T} \bar{C}\right)^{2} X H_{2} E_{1}(W)^{T}\right.} \\
& \left.+Q_{3,0}\left(\overline{X W}^{T} \bar{C}\right)^{3} X W^{T}+P_{1,2}\left(\overline{X W}^{T} \bar{B}_{1} \bar{C}\right) X_{\alpha_{2}} B_{\alpha_{2}} W^{T}\right] C=\mathrm{F} .
\end{aligned}
$$

Equation (33) and the initial conditions present nonlinear system of $(N+1)$ algebraic equations in the coefficients $c_{i}$. By solving it by using the Newton iteration method with suitable initial solution we obtain the following equation:

$$
c_{0}=\frac{1}{2}, c_{1}=1, c_{2}=\frac{1}{2}, c_{3}=0 .
$$

Therefore, the approximate solution of this example using (5) is given as follows:

$$
y_{3}(x)=\frac{1}{2} T_{0}(x)+T_{1}(x)+\frac{1}{2} T_{2}(x)=x^{2}+x,
$$

which is the exact solution of the problem (32) where

$$
\begin{aligned}
& X=\left(\begin{array}{cccc}
1 & 0 & 0 & 0 \\
1 & \frac{1}{3} & \frac{1}{9} & \frac{1}{27} \\
& \frac{2}{3} & \frac{4}{9} & \frac{8}{27} \\
1 & 1 & 1 & 1
\end{array}\right), B_{1}=\left(\begin{array}{cccc}
0 & 1 & 0 & 0 \\
0 & 0 & 2 & 0 \\
0 & 0 & 0 & 3 \\
0 & 0 & 0 & 0
\end{array}\right), W \\
& =\left(\begin{array}{cccc}
1 & 0 & 0 & 0 \\
0 & 1 & 0 & 0 \\
-1 & 0 & 2 & 0 \\
0 & -3 & 0 & 4
\end{array}\right) \\
& F=\left(\begin{array}{c}
0 \\
4.23912 \\
22.2515 \\
87.6842
\end{array}\right) \\
& X_{\alpha_{2}}=\left(\begin{array}{cccc}
0 & 0 & 0 & 0 \\
0.57735 & 0.19245 & 0.06415 & 0.0213833 \\
0.816497 & 0.544331 & 0.362887 & 0.241925 \\
1 & 1 & 1 & 1
\end{array}\right) \\
& H_{2}=\left(\begin{array}{cccc}
2 & 0 & 0 & 0 \\
0 & 4 & 0 & 0 \\
0 & 0 & 8 & 0 \\
0 & 0 & 0 & 16
\end{array}\right) \\
& B_{\alpha_{2}}=\left(\begin{array}{cccc}
0 & 0 & 2.25676 & 0 \\
0 & 0 & 0 & 4.51352 \\
0 & 0 & 0 & 0 \\
0 & 0 & 0 & 0
\end{array}\right) \\
& E_{1}=\left(\begin{array}{cccc}
1 & 1 & 1 & 1 \\
0 & 1 & 2 & 3 \\
0 & 0 & 1 & 3 \\
0 & 0 & 0 & 1
\end{array}\right), B_{v_{2}}=\left(\begin{array}{cccc}
0 & 0 & 2.22849 & 0 \\
0 & 0 & 0 & 5.14266 \\
0 & 0 & 0 & 0 \\
0 & 0 & 0 & 0
\end{array}\right), \\
& \bar{X}=\left(\begin{array}{cccccccccccccccc}
1 & 0 & 0 & 0 & 0 & 0 & 0 & 0 & 0 & 0 & 0 & 0 & 0 & 0 & 0 & 0 \\
0 & 0 & 0 & 0 & 1 & \frac{1}{3} & \frac{1}{9} & \frac{1}{27} & 0 & 0 & 0 & 0 & 0 & 0 & 0 & 0 \\
0 & 0 & 0 & 0 & 0 & 0 & 0 & 0 & 1 & \frac{2}{3} & \frac{4}{9} & \frac{8}{27} & 0 & 0 & 0 & 0 \\
0 & 0 & 0 & 0 & 0 & 0 & 0 & 0 & 0 & 0 & 0 & 0 & 1 & 1 & 1 & 1
\end{array}\right), \\
& X_{v_{2}}=\left(\begin{array}{cccc}
0 & 0 & 0 & 0 \\
0.719223 & 0.239741 & 0.0799137 & 0.0266379 \\
0.885467 & 0.590312 & 0.393541 & 0.262361 \\
1 & 1 & 1 & 1
\end{array}\right) \text {, }
\end{aligned}
$$




$$
\bar{W}^{T}=\left(\begin{array}{llllllllllllllll}
1 & 0 & -1 & 0 & 0 & 0 & 0 & 0 & 0 & 0 & 0 & 0 & 0 & 0 & 0 & 0 \\
0 & 1 & 0 & -3 & 0 & 0 & 0 & 0 & 0 & 0 & 0 & 0 & 0 & 0 & 0 & 0 \\
0 & 0 & 2 & 0 & 0 & 0 & 0 & 0 & 0 & 0 & 0 & 0 & 0 & 0 & 0 & 0 \\
0 & 0 & 0 & 4 & 0 & 0 & 0 & 0 & 0 & 0 & 0 & 0 & 0 & 0 & 0 & 0 \\
0 & 0 & 0 & 0 & 1 & 0 & -1 & 0 & 0 & 0 & 0 & 0 & 0 & 0 & 0 & 0 \\
0 & 0 & 0 & 0 & 0 & 1 & 0 & -3 & 0 & 0 & 0 & 0 & 0 & 0 & 0 & 0 \\
0 & 0 & 0 & 0 & 0 & 0 & 2 & 0 & 0 & 0 & 0 & 0 & 0 & 0 & 0 & 0 \\
0 & 0 & 0 & 0 & 0 & 0 & 0 & 4 & 0 & 0 & 0 & 0 & 0 & 0 & 0 & 0 \\
0 & 0 & 0 & 0 & 0 & 0 & 0 & 0 & 1 & 0 & -1 & 0 & 0 & 0 & 0 & 0 \\
0 & 0 & 0 & 0 & 0 & 0 & 0 & 0 & 0 & 1 & 0 & -3 & 0 & 0 & 0 & 0 \\
0 & 0 & 0 & 0 & 0 & 0 & 0 & 0 & 0 & 0 & 2 & 0 & 0 & 0 & 0 & 0 \\
0 & 0 & 0 & 0 & 0 & 0 & 0 & 0 & 0 & 0 & 0 & 4 & 0 & 0 & 0 & 0 \\
0 & 0 & 0 & 0 & 0 & 0 & 0 & 0 & 0 & 0 & 0 & 0 & 1 & 0 & -1 & 0 \\
0 & 0 & 0 & 0 & 0 & 0 & 0 & 0 & 0 & 0 & 0 & 0 & 0 & 1 & 0 & -3 \\
0 & 0 & 0 & 0 & 0 & 0 & 0 & 0 & 0 & 0 & 0 & 0 & 0 & 0 & 2 & 0 \\
0 & 0 & 0 & 0 & 0 & 0 & 0 & 0 & 0 & 0 & 0 & 0 & 0 & 0 & 0 & 4
\end{array}\right)
$$

Example 2. Consider the following nonlinear fractional delay differential equation [22, 23]:

$$
\begin{aligned}
D^{v} y(x)-y(x-0.5)+y^{3}(x)= & \frac{2}{\Gamma(1.5)} x^{0.5}-(x-0.5)^{2} \\
& -x^{6}, x \in[0,0.5]
\end{aligned}
$$

The initial conditions are $y(0)=0, y^{\prime}(0)=0$, and the exact solution is $y(x)=x^{2}$, at $v=1.5$ and $f(x)=\frac{2}{\Gamma(1.5)} x^{0.5}-(x-0.5)^{2}-x^{6}$. We apply the suggested method with $N=9$, and by the fundamental matrix equation of the problem defined in (25), we have the following equation:

$$
\left[Q_{0,2} X_{v_{2}} B_{v_{2}} W^{T}+Q_{0,0} X E_{0.5} W^{T}+Q_{2,0}\left(\overline{X W}^{T} \bar{C}\right)^{2} \cdot X W^{T}\right] C=F .
$$

Equation (36) and the subjected conditions present nonlinear system of $(N+1)$ algebraic equations in the coefficients $c_{i}$. By solving it by using the Newton iteration method with suitable initial solution, we obtain the following equation:

$$
\begin{aligned}
& c_{0}=\frac{1}{2}, c_{1}=-2.9399 \times 10^{-12}, c_{2}=\frac{1}{2}, c_{3}=-2.24153 \times 10^{-12} \\
& c_{4}=1.61475 \times 10^{-12}, c_{5}=-9.54298 \times 10^{-13}, c_{6}=4.35992 \times 10^{-13}, \\
& c_{7}=-1.45438 \times 10^{-13}, c_{8}=3.1698 \times 10^{-14}, c_{9}=-3.47446 \times 10^{-15} .
\end{aligned}
$$

therefore, the approximate solution of this example using (5) is given as follows:

$$
\begin{aligned}
y_{9}(x)= & \frac{1}{2} T_{0}(x)-2.9399 \times 10^{-12} T_{1}(x)+\frac{1}{2} T_{2}(x) \\
& +\ldots \ldots-3.47446 \times 10^{-15} T_{9}(x) .
\end{aligned}
$$

The comparison between maximum absolute errors for the proposed method and the results obtained by Haar wavelet [23] listed in Table 1.

Table 1: Comparison between maximum absolute errors for our proposed method and the results obtained by Haar wavelet [23].

\begin{tabular}{lrr}
\hline $\boldsymbol{N}$ & $\begin{array}{r}\text { Maximum absolute } \\
\text { errors of the } \\
\text { proposed method }\end{array}$ & $\begin{array}{r}\text { Maximum absolute } \\
\text { errors (Haar wavelet [23]) }\end{array}$ \\
\hline 2 & $1.585 \times 10^{-12}$ & $1.6 \times 10^{-1}$ \\
4 & $1.000 \times 10^{-12}$ & $1.5 \times 10^{-1}$ \\
8 & $5.000 \times 10^{-13}$ & $1.2 \times 10^{-1}$ \\
\hline
\end{tabular}

Example 3. Consider the following linear initial value problem [24, 25]:

$$
D^{v} y(x)+2 y^{2}\left(\frac{x}{2}\right)=1
$$

The subjected initial conditions are $y(0)=1, y^{\prime}(0)=0$, and the exact solution is given by: $y(x)=\cos (x)$ at $v=2$. The fundamental matrix equation of the problem is defined as follows:

$$
\left[Q_{0,2}(x) X_{v_{2}} B_{v_{2}} W^{T}+Q_{2,0}\left(\overline{X H}_{0.5} \bar{W}^{T} \bar{C}\right) X W^{T}\right] C=F .
$$

The approximate solution $y_{N}(x)$ introduced by the present method with $N=10$ is given as follows:

$$
C=\left[\begin{array}{ccccc}
0.76519 & -1.71389 \times 10^{-7} & -0.22980 & -1.034 \times 10^{-7} & 0.00495 \\
& -3.602 \times 10^{-8} & -0.000041 & -6.439 \times 10^{-9} & \\
& 1.9040 \times 10^{-7} & -4.315 \times 10^{-10} & -4.707 \times 10^{-10} &
\end{array}\right] .
$$


Table 2: Comparison of the absolute errors for example 3 at $v=2$.

\begin{tabular}{rrrr}
\hline $\boldsymbol{x}$ & $\begin{array}{r}\text { Present method } \\
\boldsymbol{N}=\mathbf{1 0}\end{array}$ & $\begin{array}{r}\text { Reference [24] } \\
\boldsymbol{N = 5}\end{array}$ & $\begin{array}{r}\text { Reference [25] } \\
\boldsymbol{N = \mathbf { 2 0 }}\end{array}$ \\
\hline 0.1 & $9.52 \times 10^{-13}$ & $7.41 \times 10^{-6}$ & $2.11 \times 10^{-8}$ \\
0.2 & $2.16 \times 10^{-12}$ & $1.14 \times 10^{-5}$ & $2.08 \times 10^{-8}$ \\
0.3 & $3.33 \times 10^{-12}$ & $1.34 \times 10^{-5}$ & $2.09 \times 10^{-8}$ \\
0.4 & $4.43 \times 10^{-12}$ & $1.65 \times 10^{-5}$ & $2.08 \times 10^{-8}$ \\
0.5 & $5.45 \times 10^{-12}$ & $2.07 \times 10^{-5}$ & $2.06 \times 10^{-8}$ \\
\hline
\end{tabular}

Then, the solution of the problem (39) is given as follows:

$$
\begin{aligned}
y_{10(x)}= & 0.76519 T_{0}(x)-1.71389 \times 10^{-7} T_{1}(x) \\
& -0.22980 T_{2}(x)-1.034 \times 10^{-7} T_{3}(x) \\
& +0.00495 T_{4}(x)-3.602 \times 10^{-8} T_{5}(x) \\
& -0.000041 T_{6}(x)-6.439 \times 10^{-9} T_{7}(x) \\
& +1.9040 \times 10^{-7} T_{8}(x)-4.315 \times 10^{-10} T_{9}(x) \\
& -4.707 \times 10^{-10} T_{10}(x) .
\end{aligned}
$$

Table 2 displays the comparison of the absolute errors of the present method for $N=10$ and $v=2$ with the modified Laguerre wavelets method of Iqbal et al [25] for $N=20$ and the Muntz-Legendre wavelet method of Rahimkhani et al [24] for $N=5$. We see that the present method is more accurate than the modified Laguerre wavelets and MuntzLegendre wavelet methods. The computational results for $N=10$ and different values of $v$ are given in Figure 1.

Example 4. Consider the following nonlinear fractional differential equation with argument:

$$
y^{\prime \prime}(x) D^{\alpha_{2}} y(x)+y^{2}(x) D^{\nu_{2}} y(2 x-1)+y^{\prime}(x)=f(x), x \in[0,1] .
$$

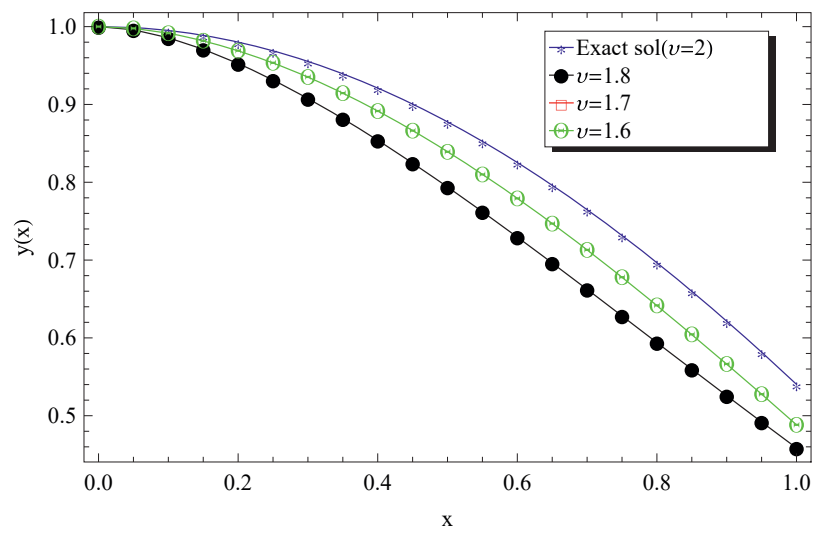

Figure 1: The numerical results of $y_{N}(x)$ for $N=10$ and $v=2,1.8,1.7$ and 1.6 for example 3.
The initial conditions are $y(0)=1, y^{\prime}(0)=1$, and the exact solution is $y(x)=x^{3}+1$ at $\alpha_{2}=1.5, v_{2}=2$ where $f(x)=27.0811 x^{2.5}+6(-1+2 x)^{2}+24(-1+2 x)\left(1+x^{3}\right)^{2}$. We apply the suggested method with $N=3$, and approximating the solution as in (5) and by the same procedure in the previous section and by using (25), we have the following equation:

$$
\begin{aligned}
& {\left[P_{2,2}\left(\overline{X W}{ }^{T} \bar{C}\right)^{2} X_{\alpha_{2}} B_{\alpha_{2}} W^{T}+Q_{2,2}\left(\overline{X W}^{T} \bar{C}\right)^{2} X_{v_{2}} B_{v_{2}} H_{2} E_{1} W^{T}\right.} \\
& \left.+P_{1,0}\left(\overline{X B W}^{T} \bar{C}\right) X_{1} B_{1} H_{3} W^{T}\right] C=\mathrm{F} .
\end{aligned}
$$

Equation (44) and the subjected initial conditions present non-linear system of $(N+1)$ algebraic equations in the coefficients $c_{i}$. By solving it by using the Newton iteration method with suitable initial solution, we obtain the following equation:

$$
c_{0}=\frac{3}{4}, c_{1}=0, c_{2}=\frac{1}{4}, c_{3}=0 .
$$

Therefore, the approximate solution of this example using (5) is given as follows:

$$
y_{4}(x)=\frac{3}{4} T_{0}(x)+\frac{1}{4} T_{2}(x)=x^{3}+1,
$$

which is the exact solution of the problem (43)

Example 5. Consider the fractional nonlinear neutral delay differential equation [26]:

$$
\begin{gathered}
y^{v}(x)-\frac{1}{2} y(x)-\frac{1}{2} y\left(\frac{x}{2}\right) y^{v}\left(\frac{x}{2}\right)=f(x), \\
0<v \leq 1, \quad-0.5 \leq x \leq 0.5,
\end{gathered}
$$

the initial conditions are $y(0)=1$, and the exact solution is $y(x)=e^{x}$ at $v=1$, where $f(x)=\frac{1}{4} e^{x}$. We apply the suggested method with $N=10$, and by approximating the solution as in (5) and by the same procedure in the previous section and by using (25), we have the following equation:

$$
\left[Q_{0,1} X_{v} B_{v} W^{T}+Q_{1,0} X W^{T}+Q_{1,1}\left(\bar{X}{\overline{H_{0.5}}}^{T} \bar{C}\right) \cdot X_{v} B_{v} H_{0.5} W^{T}\right] C=F .
$$

Equation (47) and the initial condition present non-linear system of $(N+1)$ algebraic equations in the coefficients $c_{i}$.

Table 3: Comparison of the absolute errors for example 5 at $N=9$.

\begin{tabular}{lrrr}
\hline $\boldsymbol{x}$ & Our method & HWM & Reference [26] \\
\hline 0 & $2.22 \times 10^{-16}$ & 0 & 0 \\
0.1 & $6.06 \times 10^{-12}$ & $1.33 \times 10^{-11}$ & $5.38 \times 10^{-12}$ \\
0.2 & $7.06 \times 10^{-12}$ & $3.93 \times 10^{-12}$ & $1.15 \times 10^{-11}$ \\
0.3 & $7.74 \times 10^{-12}$ & $2.84 \times 10^{-11}$ & $2.14 \times 10^{-11}$ \\
0.4 & $8.69 \times 10^{-12}$ & $1.27 \times 10^{-11}$ & $6.30 \times 10^{-12}$ \\
\hline
\end{tabular}


By solving it by using the Newton iteration method with suitable initial solution, we obtain the following equation:

$$
\begin{aligned}
& c_{0}=1.26606, c_{1}=1.13032, c_{2}=0.271493, c_{3}=0.0443388, \\
& c_{4}=0.00547301, c_{5}=0.000543584, c_{6}=0.0000446812, \\
& c_{7}=3.30673 \times 10^{-6}, c_{8}=1.68806 \times 10^{-7}, c_{9}=-1.68675 \times 10^{-8} .
\end{aligned}
$$

Therefore, the approximate solution of this example using (5) is given as follows:

$$
\begin{aligned}
y_{9}(x)= & 1.26606 T_{0}(x)+1.13032 T_{1}(x)+0.271493 T_{2}(x)+\ldots \\
& -1.68675 \times 10^{-8} T_{9}(x) .
\end{aligned}
$$

Table 3 shows the comparison of the absolute errors between the exact and approximate solutions of our method for $N=9, v=1$ by the Hermite wavelets method (HWM) and the method proposed in [26].

The comparison of the approximate values of $\mathrm{y}(\mathrm{x})$ for $N=9$ with different values of $v$ and the exact solution given in Figure 2.

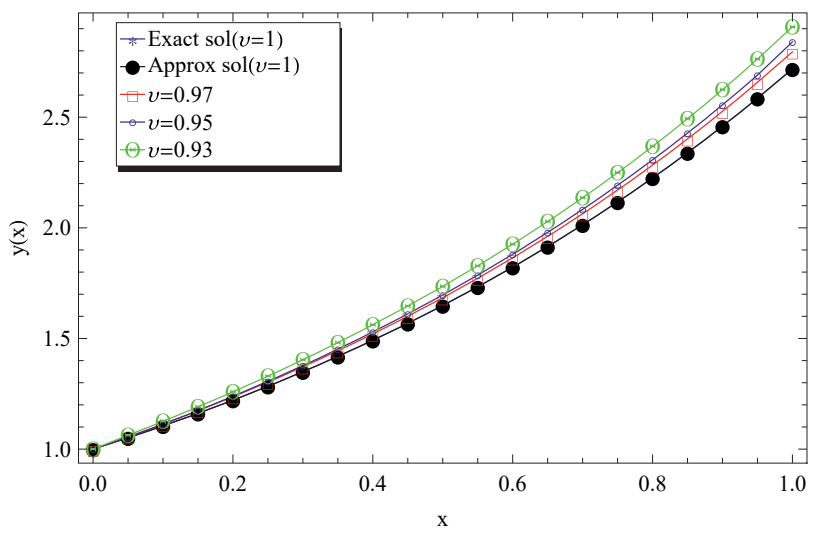

Figure 2: Comparison of $y(x)$ for $N=9$ with $v=1,0.97,0.95,0.93$ for example 5 and the exact solution.

\section{Conclusion}

In the end, we introduced a numerical study for a general form of nonlinear fractional-order differential equations with linear functional argument using Chebyshev series. In this work, we have presented a general form of the operational matrix for derivatives. The fractional-order derivatives and the ordinary operational matrix had been obtained from the proposed general form of the operational matrix for derivatives. There are no authors who have discussed this point previously in their works. We have presented many numerical examples that greatly illustrate the accuracy of the study presented to the proposed equation and also show how that the proposed method is very effective and convenient.

Author contribution: All the authors have accepted responsibility for the entire content of this submitted manuscript and approved submission.

Research funding: None declared.

Conflict of interest statement: The authors declare no conflicts of interest regarding this article.

\section{References}

[1] L. Gaul, P. Klein, and S. Kempfle, "Damping description involving fractional operators," Mech. Systems Signal Process., vol. 5, pp. 81-88, 1991.

[2] W. G. Glockle and T. F. Nonnenmacher, "A fractional calculus approach to self-similar protein dynamics," Biophys. J., vol. 68, pp. 46-53, 1995.

[3] J. H. He, "Approximate analytical solution for seepage flow with fractional derivatives in porous media, Comput," Methods Appl. Mech. Engrg., vol. 167, pp. 57-68, 1998.

[4] J. H. He, "Some applications of nonlinear fractional differential equations and their approximation," Bull. Sci. Technol., vol. 15, no. 2, pp. 86-90, 1999.

[5] R. Metzler, W. Schick, H.-G. Kilian, and T. F. Nonnenmacher, "Relaxation in filled polymers: a fractional calculus approach,"J. Chem. Phys., vol. 103, pp. 7180-7186, 1995.

[6] A. G. Ulsoy and F. M. Asl, Analysis of a System of Linear Delay Differential Equations, vol. 125, Ann Arbor MI, Mechanical Engineering Department, University of Michigan, 2003, pp. 48019-2125.

[7] C Oğuz and M Sezer, "Chelyshkov collocation method for a class of mixed functional integro-differential equations," Appl. Math. Comput., vol. 259, pp. 943-954, 2015.

[8] A. M. S. Mahdy, "Numerical studies for solving fractional integrodifferential equations," J. Ocean Eng. Sci., vol. 3, no. 2, pp. 127-132, 2018.

[9] Y. A. Amer, A. M. S. Mahdy, and E. S. M. Youssef, "Solving fractional integro-differential equations by using Sumudu transform method and Hermite Spectral Collocation Method," Comput. Mater. Con., vol. 54, no. 2, pp. 161-180, 2018.

[10] A. M. S. Mahdy, N. H. Sweilam, and M. Higazy, "Approximate solutions for solving nonlinear fractional-order smoking model," Alex. Eng. J., vol. 59, no. 2, pp. 739-752, 2020.

[11] A. Saadatmandi and M. Dehghan, "Numerical solution of the higher-order linear Fredholm integro-differential-difference equation with variable coefficients," Comput. Math. Appl., vol. 59, no. 8, pp. 2996-3004, 2010.

[12] O. K. Kürkcü, E. Aslan, and M. Sezer, "A numerical approach with error estimation to solve general integro-differential-difference equations using Dickson polynomials," Appl. Math. Comput., vol. 276, pp. 324-339, 2016.

[13] M. Gülsu, Y. Öztürk, and M. Sezer, “A new collocation method for solution of mixed linear integro-differential-difference equations," Appl. Math. Comput., vol. 216, no. 7, pp. 2183-2198, 2010. 
[14] Ş. Yüzbaşı, "Laguerre approach for solving pantograph-type Volterra integro-differential equations," Appl. Math. Comput., vol. 232, pp. 1183-1199, 2014.

[15] Ş. Yüzbaşı and N. Ismailov, “A Taylor operation method for solutions of generalized pantograph type delay differential equations,” Turk. J. Math., vol. 42, no. 2, pp. 395-406, 2018.

[16] V. lakovleva and C. J. Vanegas, "On the solution of differential equations with delayed and advanced arguments," Electron. J. Differ. Equ., vol. 13, pp. 57-63, 2005.

[17] I. A. Rus and V. A. Darzu-Ilea, "First order functional-differential equations with both advanced and retarded arguments," Fixed Point Theory, vol. 5, no. 1, pp. 103-115, 2004.

[18] Z. Liu, J. Sun and I. Szanto, “Monotone iterative technique for Riemann Liouville fractional integro-differential equations with advanced arguments," Results Math., vol. 63, no. 3-4, pp. 1277-1287, 2013.

[19] N. şahin, şYüzbaşi, and M. Sezer, “A Bessel polynomial approach for solving general linear Fredholm integro-differentialdifference equations," Int. J. Comput. Math., vol. 88, no. 14, pp. 3093-3111, 2011.

[20] K. R. Raslan, K. K. Ali, M. A. Abd El salam, and E. M. H. Mohamed, "Spectral Tau method for solving general fractional order differential equations with linear functional argument," J. Egypt. Math. Soc., vol. 27, no. 1, p. 33, 2019.
[21] K. K. Ali, M. A. Abd El Salam, and E. M. Mohamed, "Chebyshev operational matrix for solving fractional order delay-differential equations using spectral collocation method," Arab. J. Basic Appl. Sci., vol. 26, no. 1, pp. 342-353, 2019.

[22] M. M. Khader and A. S. Hendy, "The approximate and exact solutions of the fractional-order delay differential equations using Legendre seudospectral method," Int. J. Pure Appl. Math., vol. 74, no. 3, pp. 287-297, 2012.

[23] I Aziz, R Amin, and J Majak, "Numerical solution of A class of fractional delay definition equation via Haar wavelet," Appl. Math. Model., vol. 40, no. 23, 2016, https://doi.org/10.1016/j. apm.2016.07.018.

[24] P. Rahimkhani, Y. Ordokhani, and E. Babolian, “Müntz-Legendre wavelet operational matrix of fractional-order integration and its applications for solving the fractional pantograph differential equations," Numer. Algorithms, vol. 77, no. 4, pp. 1283-1305, 2017.

[25] M.A. Iqbal, U. Saeed, and S.T. Mohyud-Din, “Modified Laguerre wavelets method for delay differential equations of fractional-order," Egy. J. Basic Appl. Sci., vol. 2, pp. 50-54, 2015.

[26] U Saeed, "Hermite wavelet method for fractional delay differential equations,” J. Differ. Equ., 2014, https://doi.org/10. $1155 / 2014 / 359093$. 